\title{
Bouncing dynamics of a spring
}

\author{
M. Hubert*, F. Ludewig, S. Dorbolo, N. Vandewalle \\ GRASP, Physics Department, University of Liège, B4000 Liège, Belgium
}

\section{H I G H L I G H T S}

- We propose a model for the dynamics of a deformable bouncing object.

- We obtain the equation of motion and simulate numerically the bouncing dynamics.

- Bifurcation diagrams illustrated the influence of the deformations in the dynamics.

- The bouncing threshold curve is analyzed theoretically and numerically.

\section{A R T I C L E I N F O}

\section{Article history:}

Received 10 April 2013

Received in revised form 10 January 2014

Accepted 11 January 2014

Available online 21 January 2014

Communicated by G. Stepan

\section{Keywords:}

Non-linear dynamics

Bifurcations

Bouncing

\begin{abstract}
A B S T R A C T
We consider the dynamics of a deformable object bouncing on an oscillating plate and we propose to model its deformations. For this purpose, we use a spring linked to a damper. Elastic properties and viscous effects are taken into account. From the bouncing spring equations of motion, we emphasize the relevant parameters of the dynamics. We discuss the range of parameters in which elastic deformations do not influence the bouncing dynamics of this object and compare this behavior with the bouncing ball dynamics. By calculating the spring bouncing threshold, we evidence the effect of resonance and prove that elastic properties can make the bounce easier. This effect is for example encountered in the case of bouncing droplets. We also consider bifurcation diagrams in order to describe the consequences of a dependence on the frequency. Finally, hysteresis in the dynamics is presented.
\end{abstract}

(C) 2014 Elsevier B.V. All rights reserved.

\section{Introduction}

The bouncing ball problem is a straightforward experiment which illustrates complex deterministic dynamical systems [1]. This experiment considers a solid ball bouncing onto a rigid plate that vertically oscillates. The bouncing is possible according to two mechanisms. The first one is the impulsion provided by the plate. The driving parameter is the acceleration $\Gamma$ which measures the maximal acceleration of the plate in $g$ units. The second one is the restitution in kinetic energy at each impact. The restitution coefficient $\epsilon$ is defined as the ratio between the ball velocity after and before each impact. Despite the apparent simplicity of this model, several behaviors can be observed. For example, depending on both parameters $\Gamma$ and $\epsilon$, the bouncing ball shows bifurcations in its periodic bouncing modes, i.e. a change of periodicity in the way the ball bounces. The ball may even bounce chaotically. This system has been largely studied through past decades as reported in the articles of Luck et al. [2], Juo et al. [3] or more recently in the article of Gilet et al. [4]. Indeed, bifurcations in the bounce have been

\footnotetext{
* Corresponding author. Tel.: +3243663633.

E-mail address: maxime.hubert@ulg.ac.be (M. Hubert).

URL: http://www.grasp-lab.org (N. Vandewalle).
}

detailed and the stability of the dynamics has also been studied. Furthermore, periodicity and chaotic behaviors have been investigated. One also reports numerous applications of the bouncing ball model in various fields such as granular media [5], nanotechnology [6], neuro-sciences [7], gambling [8] or in the bouncing droplets dynamics [9].

Numerous variations have also been investigated such as the quantum version of the bouncing ball [10] or the bouncing ball on an elastic membrane [11]. This last article describes the effects of the deformations of the oscillating plate. The system has an analogue in fluid mechanics where a droplet can bounce onto an oscillating soap film [12,13]. Additional degrees of freedom have also been included in the dynamics. One can report the experiments on bouncing dimers [14] and bouncing trimers [15]. In those papers, two or three metallic beads are linked together and the formed rigid object possesses new degrees of freedom compared with the bouncing ball. As a consequence, horizontal motions and rotations of the compound object are observed.

As far as we know, a model considering the deformations of a bouncing object and their effects in the motion is still lacking. Such effects have already been observed in bouncing droplets as reported in the articles [12,16-18]. Indeed, drop deformations store potential energy thanks to surface tension effects. Thus, we propose to investigate the role of deformations by using a spring and a 
damper and studying the bounce of the compound object on a rigid and oscillating plate. In our model, the spring, at impact, stores potential energy that must be taken into account in the dynamics. Simultaneously, the damper dissipates this energy during the oscillations of the spring. Thus, those components aim to model the elastic and viscous effects of a bouncing object. Unlike the bouncing ball, this bouncing spring is not a point particle and its length varies during its motion. Furthermore, since the spring is characterized by its natural frequency, its dynamics may change with the plate frequency. In particular, the bouncing spring may resonate. This model gives us a handy way to understand the dynamics of the bouncing droplets, as explained above.

The bouncing ball and bouncing spring models will be described and compared in Section 2. In Section 3, we will determine the set of parameters that confers the bouncing spring the properties of a bouncing ball, i.e. the range of parameters in which the elastic properties of the spring are negligible. Afterwards, the elastic properties will be considered and studied through the bouncing threshold of the spring. Resonance effects will be highlighted. Some bifurcation diagrams will be provided in order to illustrate the effects of deformations. Finally, hysteresis will be described and analyzed before summarizing the work.

\section{Models}

\subsection{Bouncing ball}

The bouncing ball dynamics is the starting point of our work. The bouncing ball model is illustrated on Fig. 1(left) and considers a mass which bounces on a rigid plate oscillating sinusoidally with an angular frequency $\omega$ and an amplitude $A$. The plate vertical position $z_{p}$ is described by

$z_{p}(t)=A \cos (\omega t)$.

The ball is only submitted to gravity $g$ and to its successive interactions with the plate. Thus, in the laboratory frame, between two successive impacts, the acceleration of the ball is given by

$\frac{d^{2}\left(z_{b}-z_{p}\right)}{d t^{2}}=-g+A \omega^{2} \cos (\omega t)$,

where $z_{b}(t)$ is the height of the ball. Defining the dimensionless time $\phi=\omega t$, the dimensionless height of the ball $\alpha_{b}=z_{b} / A$ and the reduced acceleration of the plate $\Gamma=A \omega^{2} / g$, one obtains

$\frac{d^{2}\left(\alpha_{b}-\alpha_{p}\right)}{d \phi^{2}}=-\frac{1}{\Gamma}+\cos (\phi)$

One needs another equation to complete the description of the bounces of the ball. Impacts are described as follows: the speed of the ball after impact $(+)$ is linked to the speed before impact $(-)$ by

$$
\left.\frac{d\left(\alpha_{b}-\alpha_{p}\right)}{d \phi}\right|_{+}=\left.\epsilon \frac{d\left(\alpha_{b}-\alpha_{p}\right)}{d \phi}\right|_{-},
$$

where $\epsilon$ is the coefficient of restitution. Please, note that the impact is seen as instantaneous. From Eqs. (3) and (4), one observes that the dynamics is driven by only two parameters: $\Gamma$ and $\epsilon$. A more complete description of the bouncing ball dynamics is provided by Gilet et al. [4] for completely inelastic impacts.

Both parameters $\Gamma$ and $\epsilon$ characterize the mechanisms that enable the ball to bounce. The parameter $\Gamma$ is linked to the inertial effects in the system. When $\Gamma>1$ the ball takes-off only because of the motion of the plate. The parameter $\epsilon$ characterizes the impact dissipation. A non-zero value of this parameter allows the ball to leave the plate after an impact.

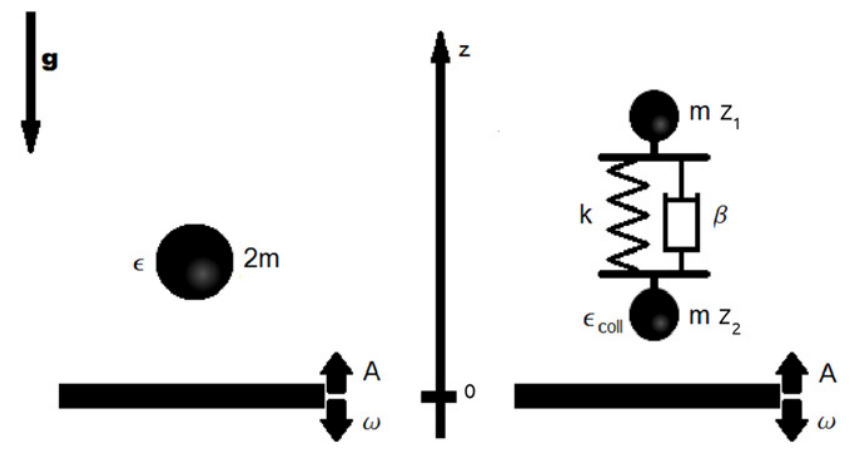

Fig. 1. Schematic representation of both models: bouncing ball (left) and bouncing spring (right). On the left, the bouncing ball model considers a point particle bouncing on a solid plate. This plate oscillates with an amplitude $A$ at angular frequency $\omega$. The impact elasticity is tuned through a parameter $\epsilon$. On the right, the bouncing spring model is represented. It consists of two identical masses $m$ linked with a spring of stiffness $k$ and a damper of viscosity $\beta$. The whole system bounces onto a solid surface oscillating sinusoidally with an amplitude $A$ and an angular frequency $\omega$. The contact between the bottom mass of the bouncing spring and the oscillating plate is tuned through the coefficient of restitution $\epsilon_{\text {coll }}$.

\subsection{Bouncing spring}

We consider that the bouncing object is vertically deformable. In order to understand this effect, we propose to model the object by two identical masses $m$ linked by a spring of stiffness $k$ and length at rest $L$. The vertical positions are respectively $z_{1}$ for the upper mass and $z_{2}$ for the lower mass. Viscous effects into the bouncing spring are modeled by a dash-pot and tuned through the dissipation parameter $\beta$. A schematic representation of the bouncing spring is provided on Fig. 1(right). Just like the bouncing ball, the compound object bounces onto a rigid surface which oscillates according to Eq. (1). One can figure out that this system is characterized by a natural frequency and thus, a deep dependence of the oscillation frequency, and resonance are expected. The motion of both masses is described by the following set of Newton's equations in the laboratory frame

$$
\left\{\begin{array}{l}
m \frac{d^{2} z_{1}}{d t^{2}}+\beta\left(\frac{d z_{1}}{d t}-\frac{d z_{2}}{d t}\right)+k\left(z_{1}-z_{2}-L\right)+m g=0, \\
m \frac{d^{2} z_{2}}{d t^{2}}-\beta\left(\frac{d z_{1}}{d t}-\frac{d z_{2}}{d t}\right)-k\left(z_{1}-z_{2}-L\right)+m g=N_{2}(t)
\end{array}\right.
$$

where $N_{2}(t)$ is the normal reaction of the surface. The shape of the normal reaction is unknown since it depends on the spring motion, but it can be evaluated through the molecular dynamics algorithm. This technique, described here-below, needs to use a restitution coefficient $\epsilon_{\text {coll }}$ in order to determine the amount of energy lost by the lower mass during impact. One has to understand that this coefficient is strictly identical to the one introduced during the study of the bouncing ball. Note that in the spring case, the contact with the plate is finite in time and not instantaneous like a bouncing ball. Defining the natural angular frequency of the spring as $\omega_{0}=\sqrt{k / m}$ and the dissipation coefficient as $\xi=\beta / 2 m \omega_{0}$, those equations can be rewritten as

$$
\left\{\begin{array}{l}
\frac{d^{2} z_{1}}{d t^{2}}+2 \xi \omega_{0}\left(\frac{d z_{1}}{d t}-\frac{d z_{2}}{d t}\right)+\omega_{0}^{2}\left(z_{1}-z_{2}-L\right)+g=0 \\
\frac{d^{2} z_{2}}{d t^{2}}-2 \xi \omega_{0}\left(\frac{d z_{1}}{d t}-\frac{d z_{2}}{d t}\right)-\omega_{0}^{2}\left(z_{1}-z_{2}-L\right)+g \\
=N_{2}(t) / m
\end{array}\right.
$$

If one introduces the dimensionless length $\alpha=z / A$, the dimensionless time $\phi=\omega t$, the dimensionless frequency $\Omega=\omega / \omega_{0}$ and the dimensionless acceleration $\Gamma=A \omega^{2} / g$, one can write

$\alpha_{p}(\phi)=\cos \phi$ 
and the set of equations ( 6 ) becomes

$$
\left\{\begin{array}{l}
\frac{d^{2} \alpha_{1}}{d \phi^{2}}+\frac{2 \xi}{\Omega}\left(\frac{d \alpha_{1}}{d \phi}-\frac{d \alpha_{2}}{d \phi}\right)+\frac{1}{\Omega^{2}}\left(\alpha_{1}-\alpha_{2}-l\right)+\frac{1}{\Gamma}=0 \\
\frac{d^{2} \alpha_{2}}{d \phi^{2}}-\frac{2 \xi}{\Omega}\left(\frac{d \alpha_{1}}{d \phi}-\frac{d \alpha_{2}}{d \phi}\right) \\
\quad-\frac{1}{\Omega^{2}}\left(\alpha_{1}-\alpha_{2}-l\right)+\frac{1}{\Gamma}=n(\phi)
\end{array}\right.
$$

where $n(\phi)$ is the dimensionless normal reaction. The dynamics is fully described by three parameters: $\Gamma$ controls the conditions of oscillation and thus the inertial effects, $\xi$ tunes the dissipation, and $\Omega$ describes the elastic effects. This last parameter has no equivalent in the bouncing ball experiment.

As previously mentioned, the shape of the normal reaction $N_{2}(t)$ is calculated thanks to a molecular dynamics algorithm. In this method, the contact between the bottom of the spring and the oscillating plate is modeled by a contact spring of stiffness $k_{p}$ and a contact damper of viscosity $\beta_{p}$. The contact spring is used for the repulsion at impact and the contact damper for the energy dissipation. Therefore, the equation for impacts is

$m_{e} \frac{d^{2} \delta}{d t^{2}}+\beta_{p} \frac{d \delta}{d t}+k_{p} \delta=0$

where $\delta$ is the interpenetration between the plate and the bottom of the bouncing spring and $m_{e}$ is the effective mass of the system, defined as $1 / m_{e}=1 / m_{p}+1 / m_{2}$ with $m_{p}$ being the mass of the plate. The latter mass could be seen as infinite since the motion of the spring does not affect the plate oscillation. The stiffness of the contact spring at impacts is chosen through the formula

$\frac{1}{2} m_{2} v_{c}^{2}=\frac{1}{2} k_{p} \delta_{c}^{2}$

which illustrates the conversion of the bottom ball kinetic energy into contact spring potential energy during impacts. In this formula, $v_{c}=A \omega$ is the characteristic speed of the system and $\delta_{c}$ is arbitrary taken as $10^{-4}$ L. From Eq. (9), one obtains the contact time between the plate and the bouncing spring [19], being

$\Delta t_{\text {coll }}=\pi \sqrt{\frac{m_{e}}{k_{p}}}\left(1-\frac{\beta_{p}^{2}}{4 m_{e} k_{p}}\right)^{-\frac{1}{2}}$.

The energy dissipation at impact can be expressed through a coefficient $\epsilon_{\text {coll }}$ (cf. Fig. 1(right)). This coefficient is given by [19]

$\epsilon_{\text {coll }}=\exp \left(-\frac{\beta_{p}}{2 m_{e}} \Delta t_{\text {coll }}\right)$.

To avoid numerical artifacts, due for example to extreme interpenetration, the time step $\Delta t_{\text {int }}$ required for the numerical integration must be linked to the collision time. A common rule to select the time step is

$\Delta t_{\text {coll }} / 100 \leq \Delta t_{\text {int }} \leq \Delta t_{\text {coll }} / 20$.

This choice is a compromise between the amount of information needed and the efficiency of the algorithm [20,21]. For every numerical result shown in this paper, the time step is fixed to $\Delta t_{\text {int }}=$ $\Delta t_{\text {coll }} / 20$. The value of $\epsilon_{\text {coll }}$ is set as closest as possible to zero in order to dissipate all kinetic energy contained in the bottom of the spring. Therefore, only the elastic properties of the spring are used for bouncing. Furthermore, setting $\epsilon_{\text {coll }} \simeq 0$ makes the bottom mass of the bouncing spring behaves likes a completely inelastic bouncing ball whose dynamics is exactly described in literature [4].

\section{Results}

\subsection{Bouncing modes}

Just like the bouncing ball, the bouncing spring bounces in different ways depending on the value of the parameters $\Gamma$ (the

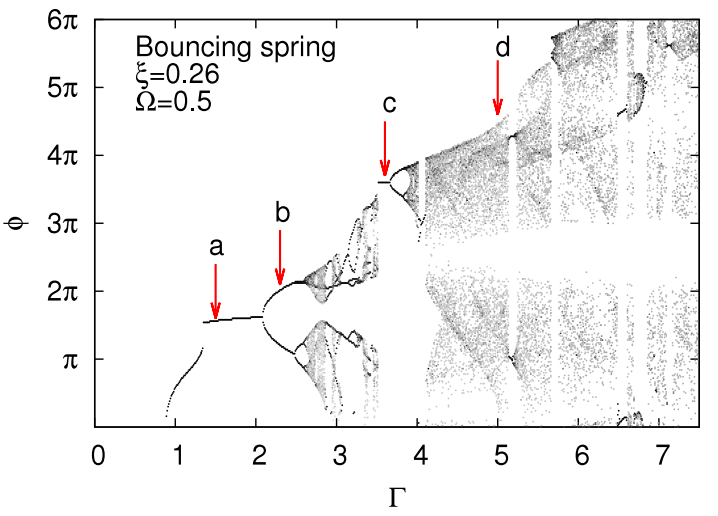

a

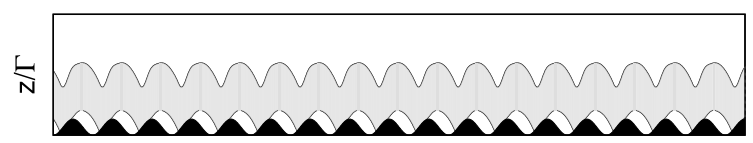

b

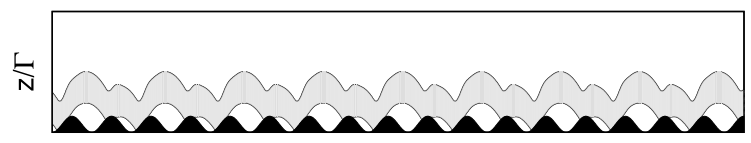

C

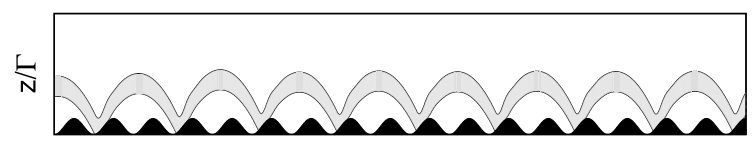

d

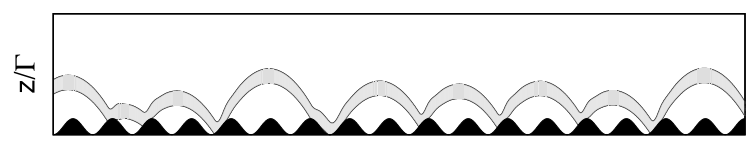

Fig. 2. Typical bifurcation diagram for the bouncing spring, the dimensionless flight time $\phi$ is shown as a function of $\Gamma$. The parameters are fixed to $\Omega=0.5$ and $\xi=0.26$. Under the bifurcation diagram are plotted four spatio-temporal diagrams which represent the spring motion (in gray) above the plate (in black). The vertical axis is normalized by $\Gamma$ in order to obtain identical oscillations of the plate. For $\Gamma=1.5$ (a), the spring follows the $(1,1)$ mode: the spring bounces once per oscillation of the plate. For $\Gamma=2.3$ (b), we observe the $(2,2)$ mode: the spring makes two distinct bounces during two oscillations of the plate. For $\Gamma=3.6$, the spring follows the $(1,2)$ mode: we observe one bounce per two oscillations of the plate. Finally, for $\Gamma=5$ (d), the motion is chaotic-like.

dimensionless acceleration), $\Omega$ (the reduced frequency) and $\xi$ (the dissipation coefficient). This could be observed on Fig. 2. This figure presents a bifurcation diagram for $\Omega=0.5$ and $\xi=0.26$. Such a diagram shows the dimensionless time of flight $\phi$ of the bouncing spring as a function of $\Gamma$. This allows us to identify bouncing modes and chaos. Different bouncing modes are observed. Some typical trajectories of the spring are represented and illustrated below the bifurcation diagram. On Fig. 2(a) $(\Gamma=1.5)$, one can observe the $(1,1)$ mode. In this mode, the spring bounces once per oscillation of the plate. On Fig. 2(b) $(\Gamma=2.3)$, the $(2,2)$ mode is illustrated. This mode is the result of a bifurcation and consists in two different bounces during two oscillations, i.e. a long parabolic flight is followed by a short one. Fig. $2(\mathrm{c})(\Gamma=3.6)$ shows the $(1,2)$ mode, the spring bounces once every two oscillations. Finally, Fig. 2(d) ( $\Gamma=5.0$ ) illustrates a chaotic behavior. The adopted bouncing mode may also be changed when $\Omega$ and $\xi$ are varied. Those bouncing modes have been encountered for elastic objects, like droplets bouncing onto liquid surfaces [18,22], rigid objects like bouncing ball [4] and bouncing droplets on elastic membranes [13].

\subsection{Bouncing ball behavior}

There is a range of parameters values in which the bouncing spring behaves like a "rigid" object, like a ball. Indeed, the bouncing 
spring is seen as "rigid" if it is able to completely damp its oscillations between two successive impacts on the surface. In this case, no potential energy is stored during its flights and its elastic properties have no effect in its subsequent dynamics. In order to obtain a criterion to measure the proximity to the bouncing ball behavior, we have to compare the characteristic time of damping $\tau_{\text {damp }}$ and the characteristic time between impacts $\tau_{\text {imp }}$. The first one, $\tau_{\text {damp }}$, is given through the set of Eqs. (6). Let us consider an in-flight spring, the equation governing its elongation is

$\frac{d^{2} \Delta z}{d t^{2}}+4 \xi \omega_{0} \frac{d \Delta z}{d t}+2 \omega_{0}^{2}(\Delta z-L)=0$

where $\Delta z=z_{1}-z_{2}$. The integration of this equation provides the following solution

$$
\begin{aligned}
\Delta z(t)= & \exp \left(-2 \xi \omega_{0} t\right)\left[A \exp \left(\sqrt{2} \omega_{0} \sqrt{2 \xi^{2}-1} t\right)\right. \\
& \left.+B \exp \left(-\sqrt{2} \omega_{0} \sqrt{2 \xi^{2}-1} t\right)\right]+L
\end{aligned}
$$

where $A$ and $B$ are two constants which depend on the initial conditions. From this solution, one obtains the characteristic damping time $\tau_{\text {damp }} \simeq 1 / \omega_{0} \xi$. The characteristic time between impacts is given by $\tau_{\text {imp }} \simeq 1 / \omega$, since one supposes an impact per oscillation of the plate. We introduce the dimensionless number $\Xi$ named rigidity of the spring, defined by the ratio

$\Xi=\frac{\tau_{\text {imp }}}{\tau_{\text {damp }}}=\frac{\xi}{\Omega}$.

When $\tau_{\text {imp }} \ll \tau_{\text {damp }}$, one obtains $\Xi \ll 1$, the spring does not have the time to dissipate the potential energy it contains and this storage influences the next rebound. This case will be discussed in the following section. In the limit $\Xi \gg 1, \tau_{\text {imp }} \gg \tau_{\text {damp }}$, the spring returns to equilibrium without storing any potential energy into deformation, the spring length is always equals to $L$ when the spring touches the plate. In this case, the bouncing spring acts like a nondeformable rigid ball. Indeed, the set of Eqs. (8) can be rewritten as follows. Between two impacts, one has

$$
\left\{\begin{array}{l}
\frac{d^{2} \alpha_{c}}{d \phi^{2}}+\frac{1}{\Gamma}=0 \\
\frac{d^{2} \Delta \alpha}{d \phi^{2}}+4 \Xi \frac{d \Delta \alpha}{d \phi}+2 \frac{\Xi^{2}}{\xi^{2}}(\Delta \alpha-l)=0,
\end{array}\right.
$$

where the first equation describes the motion of the spring center through $\alpha_{c}=\left(\alpha_{1}+\alpha_{2}\right) / 2$ and the second one describes the elongation of the spring $\Delta \alpha=\alpha_{1}-\alpha_{2}$. In the limit $\Xi \gg 1$, the inertial term $d^{2} \Delta \alpha / d \phi^{2}$ can be neglected. Thus, one obtains after integration

$$
\left\{\begin{array}{l}
\frac{d^{2} \alpha_{c}}{d \phi^{2}}+\frac{1}{\Gamma}=0 \\
\Delta \alpha(\phi) \simeq l
\end{array}\right.
$$

Therefore, the spring behaves like a ball of reduced diameter $l$ submitted to gravity between two impacts. This behavior is strictly equivalent to the bouncing ball dynamics. In the case of the rigid bouncing spring, only two parameters remain: the reduced acceleration $\Gamma$ and the viscosity $\xi$. Since the effects of elasticity are lost, the parameter $\Omega$ vanishes. Furthermore, one can understand that the viscosity $\xi$ may act like a kind of restitution coefficient $\epsilon$ in for high $\Xi$ number, i.e. $\epsilon=f(\xi)$ when $\Xi \gg 1$. The equivalence between the dynamics of the spring for high $\Xi$ number and the dynamics of the completely inelastic ball is presented in Fig. 3 for $\Xi=650$ and $\epsilon=0$. This figure compares the bifurcation diagrams of both objects. Both diagrams are identical. The modes adopted by the rigid bouncing spring during its motion are the same to those used by the bouncing ball. Those diagrams are fully described in Gilet et al. [4].

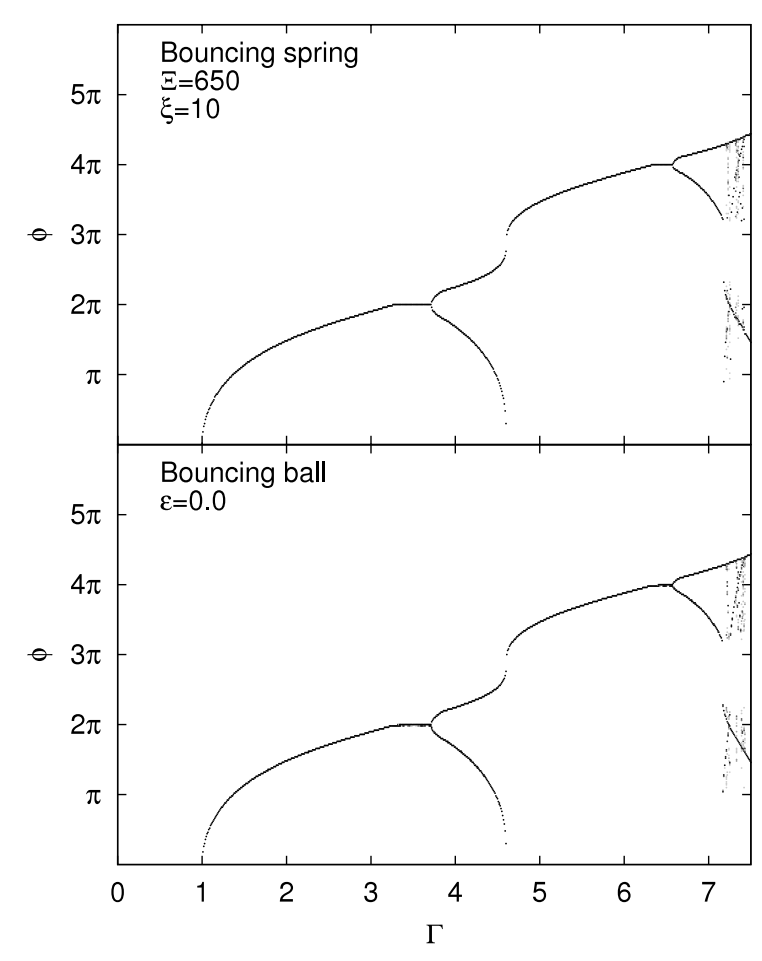

Fig. 3. Comparison between the rigid bouncing spring $(\Xi=650, \xi=10)$ and the completely inelastic bouncing ball $(\epsilon=0)$. Bouncing modes adopted by both objects are similar and the diagrams cannot be distinguished from each other.

The dynamics of the partially elastic ball can also be reproduced by changing the spring viscosity for high $\Xi$ number. Indeed, if the bouncing spring remains rigid, its elastic properties are useless. But, if the viscosity is decreased, less energy is lost during the squeezing of the spring at impacts. This could be seen as a partially elastic collision. Thus, in this case, the bouncing spring behaves like a partially elastic bouncing ball. An example of the equivalence between the dynamics of the rigid low-viscosity spring and the partially elastic ball is given in Fig. 4.

According to the observations above, the elastic properties of a bouncing object does not become apparent for high $\Xi$ number. The dynamics of this bouncing object is exactly described by the bouncing ball model. Indeed, in the case of the rigid bouncing spring, two parameters remain to describe the dynamics: the reduced acceleration $\Gamma$ and the viscosity $\xi$. This last parameter plays the role of a kind of restitution coefficient $\epsilon$. Note also that the dependence of the bouncing spring dynamics toward $\Omega$ vanishes in the case of a rigid bouncing spring.

\subsection{Deformations}

\subsubsection{Bouncing threshold}

For $\Xi<1$, the spring is significantly constrained by the plate motion. It keeps some potential energy in its deformations between two successive impacts and this elastic storage deeply influences the bouncing dynamics. Depending on the value of the dimensionless frequency $\Omega$, the energy storage is different, since resonance can be involved. Thus, the effects of three parameters $\Gamma, \Omega$ and $\xi$ have to be taken into account. As a consequence, 3D diagrams have to be considered in order to describe the bouncing spring dynamics. The axis of those diagrams are the $\Gamma$ and $\phi$ axis already observed for the bouncing ball, but also the $\Omega$ axis which describes the elastic properties of the bouncing spring.

A handy way to identify the effects of frequency on the motion is to draw the bouncing threshold of the spring, i.e. starting with a spring at rest on the plate, which value of $\Gamma$ is required to observe 


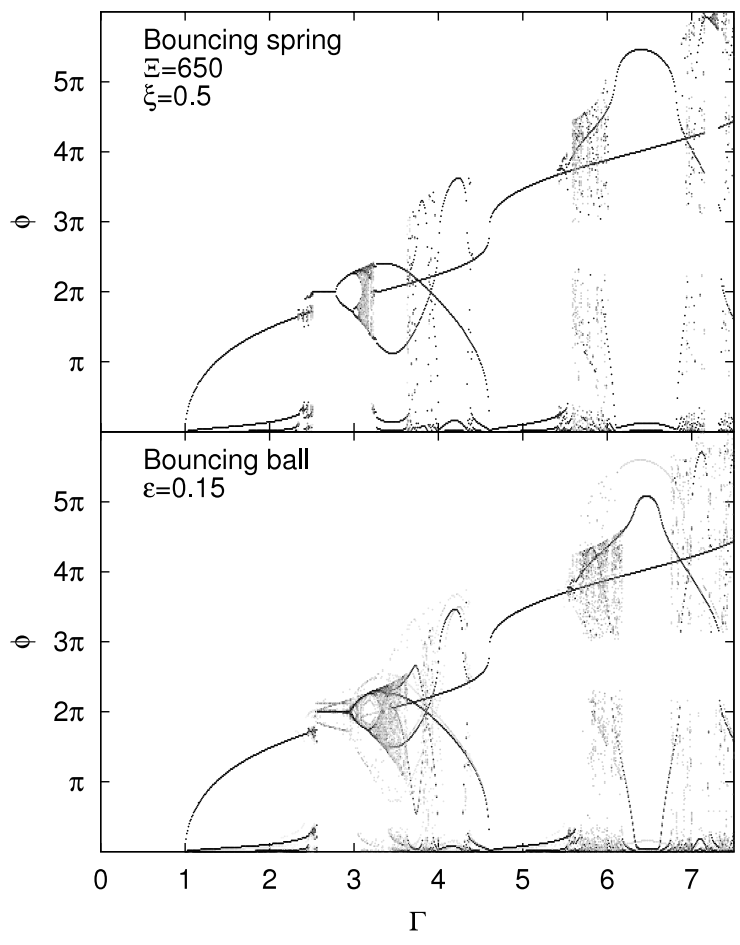

Fig. 4. Comparison between the rigid low-viscosity bouncing spring $(E=$ $650, \xi=0.5)$ and the partially elastic bouncing ball $(\epsilon=0.15)$. We observe that with a lower viscosity, the spring behaves like a partially elastic ball. Only small differences are seen between both diagrams. In particular, forbidden zones exist in both diagrams.

a take-off? This approach is the one used by Eichwald [11] or by Gilet [4] in the case of deformable plates. Furthermore, in so doing, one can study the coupled effects of both the frequency and inertia in a two dimensional diagram. In order to build those diagrams, let us start with a spring at rest on the oscillating plate. The lower mass will follow the plate motion

$\alpha_{2}(\phi)=\cos \phi$.

And the upper mass is assumed to follow the equation of motion

$\alpha_{1}(\phi)=\alpha^{\prime} \cos (\phi+\theta)$

which means that the mass $m_{1}$ oscillates at the plate frequency but its motion is eventually phase-shifted with a phase $\theta$. The amplitude of this motion is $\alpha^{\prime}$. The minimal value of $\Gamma$ required to takeoff, $\Gamma_{\text {th }}$, is provided by the set of Eqs. (8). Substituting $\alpha_{1}$ and $\alpha_{2}$ by the expression above, one obtains, with the first equation of ( 8 )

$\alpha^{\prime}=\sqrt{\frac{1+(2 \xi \Omega)^{2}}{\left(1-\Omega^{2}\right)^{2}+(2 \xi \Omega)^{2}}}$,

$\theta=-\operatorname{acos}\left(\sqrt{\frac{\left[\left(1-\Omega^{2}\right)+(2 \xi \Omega)^{2}\right]^{2}}{\left[1+(2 \xi \Omega)^{2}\right]\left[\left(1-\Omega^{2}\right)^{2}+(2 \xi \Omega)^{2}\right]}}\right)$.

Those expressions give the upper mass amplitude of motion and phase-shift in terms of $\Omega$ and $\xi$, respectively the dimensionless frequency and dissipation coefficient. The second equation of the set (8) gives the threshold. Indeed, one remarks that the moment when the spring takes-off is characterized by $n_{2}\left(\phi_{0}\right)=0$ where $\phi_{0}$ is the take-off instant. But, at the same time, both masses still follow Eqs. (19) and (20). Thus, once those assumptions injecting in the second equation of (8), one obtains

$\cos \phi_{0}\left(\alpha^{\prime} \cos \theta+1\right)-\alpha^{\prime} \sin \phi_{0} \sin \theta=\frac{2}{\Gamma}$.

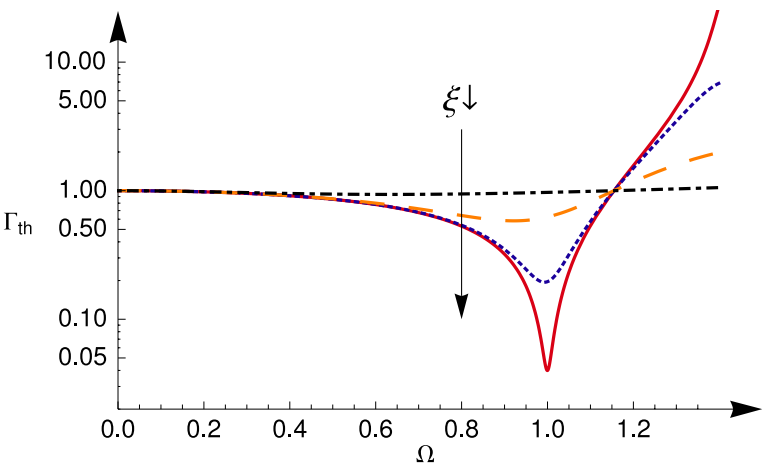

Fig. 5. Bouncing threshold $\Gamma_{\text {th }}$ as a function of $\Omega$ for several viscosities $(\xi=0.01$, $0.05,0.2,1)$. One can see that a minimum appears near $\Omega=1$ when the viscosity is decreased. At this frequency, the spring resonates, absorbs a large amount of potential energy, and is able to maintain its bounce for low values of $\Gamma$. For $\xi=1$, the threshold remains constant and equals to $\Gamma_{\text {th }}=1$. This is the same behavior than the bouncing ball. Indeed, for this curve, the spring is characterized by $\xi>1$ and thus behaves like a ball. When the frequency is increased, the threshold is slightly higher than one, the spring leaves the rigid limit. Thus, the deformations start to influence the bounce.

A minimal value of $\Gamma$ leads to a maximal value of the left term of this relation. Canceling the derivative of this term with respect to $\phi$ gives the take-off instant at $\Gamma_{\text {th }}$

$\phi_{0}=\arctan \left(\frac{2 \xi \Omega^{3}}{2+\left(8 \xi^{2}-3\right) \Omega^{2}+\Omega^{4}}\right)$.

Then, the bouncing threshold $\Gamma_{\mathrm{th}}(\Omega)$ is given by

$\Gamma_{\text {th }}(\Omega)=2 \sqrt{\frac{\left(1-\Omega^{2}\right)^{2}+4 \xi^{2} \Omega^{2}}{\left(2-\Omega^{2}\right)^{2}+16 \xi^{2} \Omega^{2}}}$.

This equation is plotted on Fig. 5 for $\xi=0.01, \xi=0.05, \xi=$ 0.2 and $\xi=1$. One sees that a minimum appears and moves to $\Omega=1$ when the spring dissipation coefficient $\xi$ is decreased. The spring resonates, stores a large amount of potential energy that it uses to take-off. As a consequence, the spring is able to maintain the bounce for low values of the dimensionless acceleration $\Gamma$. For high dissipation coefficients $(\xi>1)$, the minimum vanishes because the spring cannot resonate. In this case, the bouncing threshold remains constant with $\Gamma_{\text {th }}=1$. One can understand this by calculating the spring rigidity $\Xi$ : for low values of $\Omega$ and high viscosities $\xi$, the spring is rigid and acts like a bouncing ball for which $\Gamma_{\text {th }}=1$. Nevertheless, the bouncing threshold increases slightly because, when the frequency is increased, the spring reaches lower values of $\Xi$ and no longer behaves like a bouncing ball.

In summary, the excitation frequency deeply influences the bouncing spring dynamics when $\Xi<1$. In particular, resonance makes the bouncing possible even for $\Gamma<1$. This behavior strongly contrasts with the bouncing ball dynamics where $\Gamma_{\text {th }}$ remains equal to one for every frequencies. Furthermore, the less the dissipation, the more important the effect of frequency. Indeed, for low values of $\xi$, the spring keep a large amount of potential energy between two impacts.

\subsubsection{Bifurcation diagrams}

In order to characterize the bouncing when the deformations are relevant, let us consider the $(\Gamma, \phi)$ and $(\Omega, \phi)$ bifurcation diagrams. Those ones represent sections of the 3D bifurcation diagrams for which $\Gamma$ or $\Omega$ are kept constant. For this purpose, we propose to analyze three different situations (below, at and above resonance) for a bouncing spring that is allowed to resonate, one arbitrary chooses $\xi=0.05$.

Knowing that $\Gamma$ tunes the inertia in the system, the $(\Gamma, \phi) \mathrm{di}-$ agrams are used to characterize inertial effects in the bouncing dynamics without considering the effects of frequency. However, 


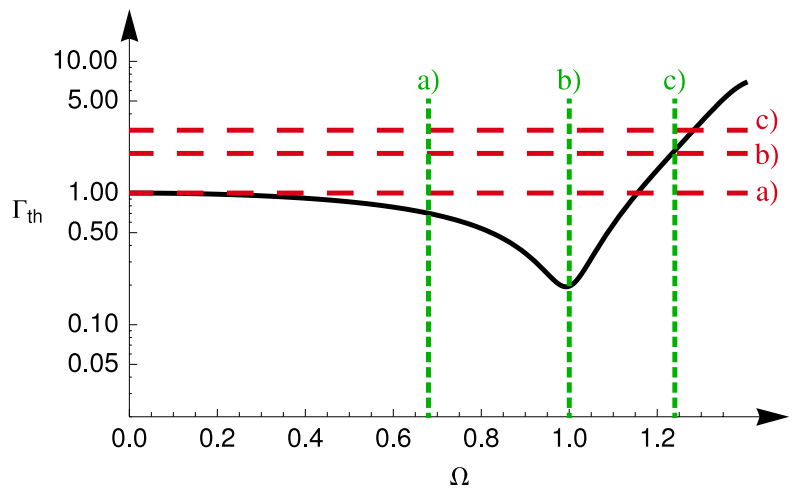

Fig. 6. Bouncing threshold (curve in plain black) and representations of the sections in the 3D bifurcation diagram for a bouncing spring with $\xi=0.05$. In dashed red (horizontal lines), the $(\Gamma, \phi)$ diagrams and, in dotted green (vertical lines), the $(\Omega, \phi)$ diagrams. The spring dynamics in those sections are illustrated in the diagrams of Figs. 7 and 8.

numerous $(\Gamma, \phi)$ diagrams acquired for several values of $\Omega$ can give a valuable information about the influence of deformations in the dynamics. The bouncing threshold, in this case, is visible on Fig. 6 . We consider dimensionless frequencies below and beyond resonance (resp. $\Omega=0.67$ and $\Omega=1.24$ ) but also at resonance ( $\Omega=1.00$ ). We observe that the spring behaves differently depending on the value of $\Omega$. At resonance, the dynamics shows numerous bifurcations that lead to chaotic behaviors for $\Gamma \simeq 2$ and $\Gamma \simeq 4$. The complexity of the diagram at resonance, in comparison with the others, may come from the transfer of energy form the plate to the spring at this frequency. Beyond resonance $(\Omega=$ 1.24 ), no chaotic behavior can be seen. Below resonance ( $\Omega=$ $0.67)$, the bouncing spring follows a $(1,1)$ mode in a large interval of $\Gamma$, chaotic behavior appears beyond $\Gamma \simeq 3$. Finally, one observes that the times of flight decrease when the frequency increases.

Let us now consider the $(\Omega, \phi)$ diagrams. Because $\Omega$ is linked to the spring elastic properties, those diagrams are used to visualize their effects on the dynamics. Furthermore, they are specific to the bouncing spring since the bouncing ball is not subject to any frequency effect. Thus, the differences with the bouncing ball dynamics is clearly shown with those diagrams. Some examples of those diagrams are provided on Fig. 8 for $\Gamma=1,2$ and 3 and $\xi=0.05$. The corresponding threshold is also visible of Fig. 6 . Bifurcations could be observed while $\Omega$ decreases and lead to a chaotic motion for low values of the reduced frequency. This behavior can be explained as follows: the energy of the oscillating plate is proportional to $A^{2} \Omega^{2} \propto \Gamma^{2} / \Omega^{2}$. Thus, if $\Gamma$ is maintained constant, decreasing $\Omega$ increases the energy of the plate, this energy being stored into the spring at impacts. One can understand that diminishing the value of $\Omega$ has the same effect on the bifurcation diagrams than increasing the value of $\Gamma$ : more energy can be transferred to the spring that performs longer flights. This transfer also explains the shrinking of the diagrams, observed in the $(\Gamma, \phi)$ diagrams above, when $\Omega$ increases. However, there is no direct link between the spring resonance and the length of its flights.

In summary, in order to characterize the effects of frequency, the bouncing threshold of the spring has been calculated. The obtained formula shows a minimum due to the resonance of the spring. For this frequency, the energy transferred from the plate to the spring is maximal and is stored in its deformations. This behavior explains the minimum. One observes that the bouncing threshold is significantly modified by the dissipation within the spring. When the dissipation is increased, the effects of frequency disappears. In particular, when the coefficient $\xi$ is high, the spring is characterized by $\Xi>1$ and behaves like a bouncing ball for which $\Gamma_{\text {th }}=1$. Because the information about the time of flight are lost in the bouncing threshold diagram, $(\Gamma, \phi)$ and $(\Omega, \phi)$ diagrams have been built. An observation can be made about the influence of the frequency of the flight time: the dimensionless flight

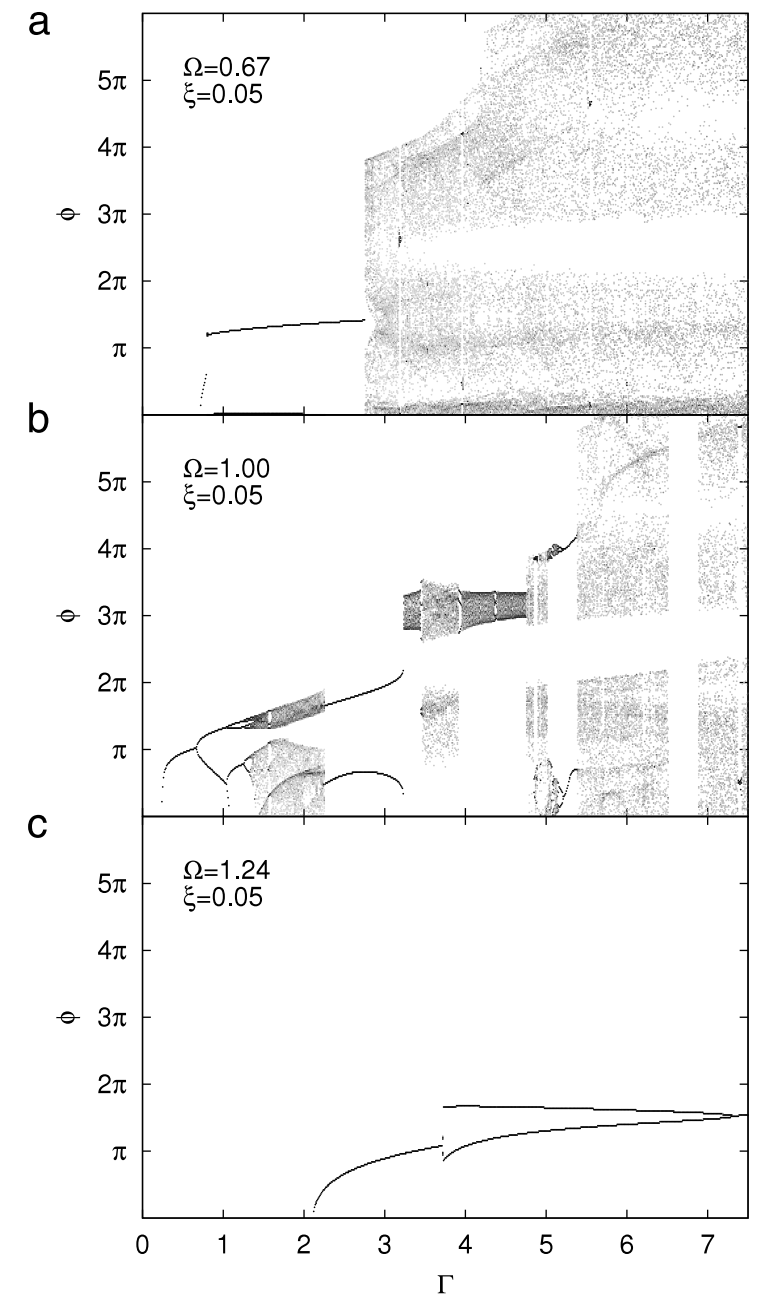

Fig. 7. Comparison between three $(\Gamma, \phi)$ diagrams made for (a) $\Omega=0.67$ (below resonance), (b) $\Omega=1.00$ (at resonance) and (c) $\Omega=1.24$ (beyond resonance) respectively. The dissipation coefficient is fixed to $\xi=0.05$ for every plots. One observes some forbidden regions already seen in the bouncing ball dynamics in Figs. 3 and 4. In particular, the diagrams press down when $\Omega$ increases.

time decreases when the frequency increases. This effect finds its source in the energy transferred to the plate. Indeed, the higher the frequency, the less the energy transferred. Nevertheless, the dependence of $\Omega$ is difficult to characterize. The resonance of the spring seems to have no effect on the flight time, on the possible bifurcations in the diagrams or even on the appearance of chaotic behavior.

\subsection{Hysteresis}

For $\Xi<1$, the bouncing spring is subject to hysteresis. Indeed, depending on the way $\Gamma$ (or $\Omega$ ) varies during an experiment, the result can be different. This fact is illustrated in Fig. 9 for a spring with $\xi=0.05$. The curve in gray corresponds to an acquisition for increased values of $\Gamma$. This is the case one studied in Section 3.3.1. Indeed, the spring starts its motion at rest on the immobile plate. The plate starts to oscillate, and the value of $\Gamma$ for which the spring bounces is plotted on the graph. For the second curve, the spring begins its motion on a plate oscillating with a large amplitude. The value of the dimensionless acceleration is decreased and the value of $\Gamma$ for which the spring sticks permanently to the plate is plotted. One observes that the curves do not collapse on each other. Indeed, for the second curve, several minima appear. Those are not explained by Eq. (25). As a consequence, in the case of decreased values of $\Gamma$, the bounce can be sustained for lower values of 


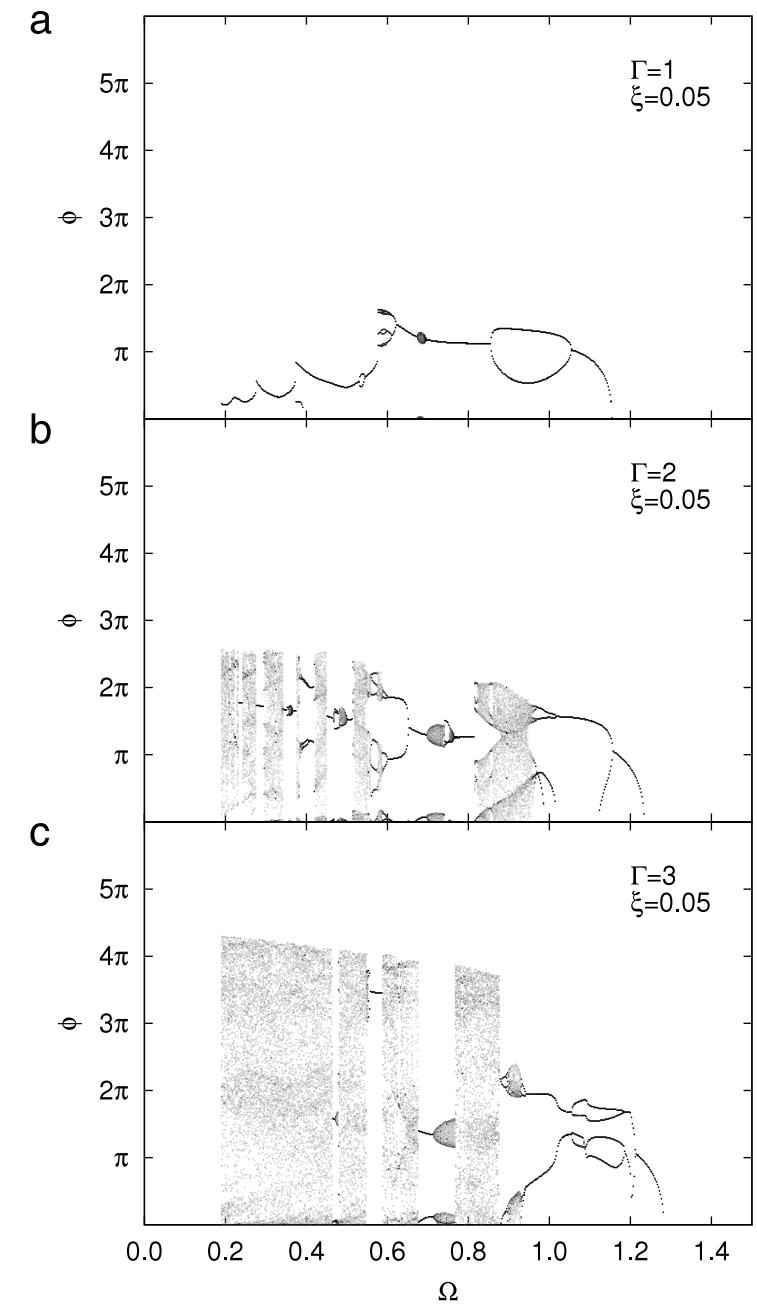

Fig. 8. Comparison between three $(\Omega, \phi)$ diagrams made for (a) $\Gamma=1$, (b) $\Gamma=2$ and (c) $\Gamma=3$ respectively with $\xi=0.05$. One observes the effect of the reduced frequency in the dynamics of the spring. In particular, the spring can bounce below $\Gamma=1$ due to some resonance of the spring. Contrarily to the $(\Gamma, \phi)$ diagrams, the flight time increases for decreasing $\Omega$ and the bifurcation cascades appear for low values of $\Omega$.

the dimensionless acceleration. This behavior has already been observed in other deformable systems such as the bouncing droplet dynamics [17]. Nevertheless, this behavior is still misunderstood. Please note that every diagrams presented before are acquired for increased value of $\Gamma$ or $\Omega$.

\section{Conclusion}

We have proposed the bouncing spring model: two masses linked together with a spring and a damper, the compound object bouncing onto a rigid and oscillating plate. The spring aims to model the possible deformations of a bouncing object while the damper dissipates the energy inherent to such deformations. We show the dependence in three parameters, each of them controlling the three essential mechanisms: the inertia through the parameter $\Gamma$, the dissipation through $\xi$ and the elastic effects through $\Omega$. We also point out that for a given range of parameters, the bouncing spring behaves like a bouncing ball. We define a parameter $\Xi=\xi / \Omega$ to measure the proximity with the bouncing ball dynamics. If $\Xi>1$, the bouncing spring behaves like a bouncing ball and if $\Xi<1$, we have to consider the elastic properties of the spring. In order to characterize the effects of deformations, we choose to study the spring bouncing threshold and obtain its analytic expression. In particular, we prove that a resonance makes

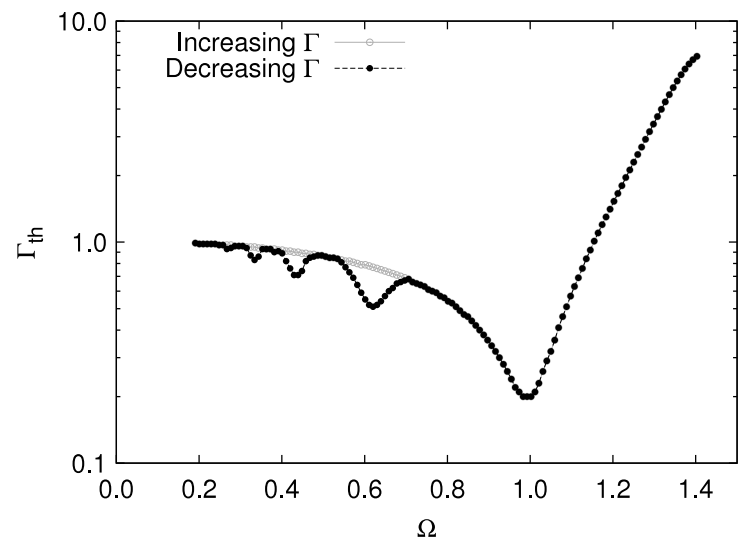

Fig. 9. Numerical acquisition of the bouncing threshold. In gray, $\Gamma$ is increased during the experiment. In black, $\Gamma$ is decreased. One observes that several minima appear when $\Gamma$ is decreased.

the bounce easier. We also show that the effects of resonance vanish when the dissipation is increased and, when $\xi>1$, completely disappear. Nevertheless, a deep study on the motion through bifurcation diagrams shows that the resonance has no visible effects on the spring flight time nor in the appearance of bifurcations or chaos. Finally, we point out the existence of hysteresis in the dynamics reported in other deformable bouncing systems.

\section{Acknowledgments}

SD thanks FNRS (Brussels, Belgium) for financial support. This work is also supported by the Morecar T-REX project (Feder, Wallonia).

\section{References}

[1] N. Tufillaro, T. Abbott, J. Reilly, An Experimental Approach to Nonlinear Dynamics and Chaos, Addison-Wesley, 1992.

[2] J. Luck, A. Mehta, Bouncing ball with a finite restitution: chattering, locking and chaos, Phys. Rev. E 48 (1993) 3988.

[3] A.C.J. Juo, R.P.S. Han, The dynamics of a bouncing ball with a sinusoidally vibrated table revisited, Nonlinear Dynam. 10 (1996) 1.

[4] T. Gilet, N. Vandewalle, S. Dorbolo, Completely inelastic ball, Phys. Rev. E 79 (2009) 055201(R).

[5] J. Pastor, D. Maza, I. Zuriguel, A. Garcimartin, J.-F. Boudet, Time resolved particule dynamics in granular convection, Physica D 232 (2007) 128.

[6] N. Burnham, A. Kulik, G. Gremaud, Nanosubharmonics: the dynamics of small nonlinear contacts, Phys. Rev. Lett. 74 (1995) 5092.

[7] R. Ronsse, P. Lefèvre, R. Sepulchre, Robotics and neuroscience: a rhythmic interaction, Neural Netw. 21 (2008) 577.

[8] M. Small, C. Tse, Predicting the outcome of roulette, Chaos 22 (2012) 033150.

[9] Y. Couder, E. Fort, C.-H. Gauthier, A. Boudaoud, From bouncing to floating: noncoalescence of drops on a fluid bath, Phys. Rev. Lett. 94 (2005) 177801.

[10] J. Gea-Banacloche, A quantum bouncing ball, Amer. J. Phys. 67 (1999) 776.

[11] B. Eichwald, M. Argentina, X. Noblin, F. Celestini, Dynamics of a ball bouncing on a vibrated elastic membrane, Phys. Rev. E 82 (2010) 016203.

[12] T. Gilet, J.W.M. Bush, Chaotic bouncing of a droplet on a soap film, Phys. Rev. Lett. 102 (2009) 014501.

[13] T. Gilet, J.W.M. Bush, The fluid trampoline: droplets bouncing on a soap film, J. Fluid Mech. 625 (2009) 167.

[14] S. Dorbolo, D. Volfson, L. Tsimring, A. Kudrolli, Dynamics of a bouncing dimer, Phys. Rev. Lett. 95 (2005) 044101.

[15] S. Dorbolo, F. Ludewig, N. Vandewalle, Bouncing trimer: a random selfpropelled particle, chaos and periodical motions, New J. Phys. 11 (2009) 033016.

[16] S. Dorbolo, D. Terwagne, N. Vandewalle, T. Gilet, Resonant and rolling droplet, New J. Phys. 10 (2008) 113021

[17] T. Gilet, D. Terwagne, N. Vandewalle, S. Dorbolo, Dynamics of a bouncing droplet onto a vertically vibrated interface, Phys. Rev. Lett. 100 (2008) 167802.

[18] D. Terwagne, F. Ludewig, N. Vandewalle, S. Dorbolo, The role of the droplet deformations in the bouncing droplet dynamics, Phys. Fluids (2013) 122101.

[19] J. Schäfer, S. Dippel, D.E. Wolf, Force schemes in simulations of granular materials, J. Physique I 6 (1996) 5.

[20] L.E. Silbert, D. Ertas, G.S. Grest, T.C. Halsey, D. Levine, S.J. Plimpton, Granular flow down an inclined plane: bagnold scaling and rheology, Phys. Rev. E 64 (2001) 051302.

[21] L.E. Silbert, D. Ertas, G.S. Grest, T.C. Halsey, D. Levine, Geometry of frictionless and frictional sphere packings, Phys. Rev. E 65 (2002) 031304.

[22] S. Protière, A. Boudaoud, Y. Couder, Particle-wave association on a fluid interface, J. Fluid Mech. 554 (2006) 85. 